\title{
Four-phase patterns in forced oscillatory systems
}

\author{
A. L. Lin, ${ }^{1}$ A. Hagberg, ${ }^{2,}$ 用 A. Ardelea, ${ }^{3}$ M. Bertram, ${ }^{1}$ H. L. Swinney, ${ }^{1}$ and E. Meron ${ }^{4}$, \\ ${ }^{1}$ Center for Nonlinear Dynamics and Department of Physics, The University of Texas at Austin, Austin, TX 78712 \\ ${ }^{2}$ Center for Nonlinear Studies and T-7, Theoretical Division, \\ Los Alamos National Laboratory, Los Alamos, NM 87545 \\ ${ }^{3}$ CFD Laboratory and ASE/ME Department, The University of Texas at Austin, Austin, TX 78712 \\ ${ }^{4}$ Department of Energy and Environmental Physics, BIDR, and Physics Department, \\ Ben-Gurion University, Sede Boker Campus 84990, Israel
}

(Received 23 February 2000)

\begin{abstract}
We investigate pattern formation in self-oscillating systems forced by an external periodic perturbation. Experimental observations and numerical studies of reaction-diffusion systems and an analysis of an amplitude equation are presented. The oscillations in each of these systems entrain to rational multiples of the perturbation frequency for certain values of the forcing frequency and amplitude. We focus on the subharmonic resonant case where the system locks at one fourth the driving frequency, and four-phase rotating spiral patterns are observed at low forcing amplitudes. The spiral patterns are studied using an amplitude equation for periodically forced oscillating systems. The analysis predicts a bifurcation (with increasing forcing) from rotating four-phase spirals to standing two-phase patterns. This bifurcation is also found in periodically forced reaction-diffusion equations, the FitzHugh-Nagumo and Brusselator models, even far from the onset of oscillations where the amplitude equation analysis is not strictly valid. In a Belousov-Zhabotinsky chemical system periodically forced with light we also observe four-phase rotating spiral wave patterns. However, we have not observed the transition to standing two-phase patterns, possibly because with increasing light intensity the reaction kinetics become excitable rather than oscillatory.
\end{abstract}

\section{INTRODUCTION}

Spatially extended systems characterized by the coexistence of two or more stable states compose a broad class of nonequilibrium pattern forming systems. The most common multistable systems are those that exhibit bistability, (e.g. chemical systems [1], 2], vertically vibrated granular systems [3], and binary fluid convection [4]). Spatial patterns in these systems involve alternating domains of the two different stable states, which are separated from each other by interfaces or fronts. Bistable systems support a variety of patterns from spiral waves to splitting spots and labyrinths [5, 6, 77, 8, 9]. In some systems, such as the ferrocyanide-iodate-sulfite reaction [6, 10] and the oxidation of carbon monoxide on a platinum surface [11], the bistability arises from the nonlinear nature of the system. In other systems such as liquid crystals in a rotating magnetic field [12, 13, 14] and periodically forced oscillators [15], the bistability arises from a broken symmetry.

Periodically forced oscillatory systems are convenient systems for exploring multistability in pattern formation since the number of coexisting stable states can be controlled by changing the forcing frequency. Applying a periodic force of sufficient amplitude and at a frequency $\omega_{f} \approx \frac{n}{m} \omega_{0}$, where $\omega_{0}$ is the oscillation frequency of the unforced system, entrains the system to the forcing frequency. The entrained system has $n$ stable states each with the same oscillation frequency but in one of $n$ oscillation phases separated by multiples of $2 \pi / n$. We refer to the $n$ different phase shifted states as "phase states" of the system.

Recent experiments using the ruthenium-catalyzed Belousov-Zhabotinsky reaction forced by periodic illumination revealed subharmonic resonance regimes $\omega_{f}: \omega_{0}=2: 1$, $3: 2,3: 1,4: 1$, with two $(2: 1)$, three $(3: 2,3: 1)$, and four $(4: 1)$ stable phase states [2, 16. Patterns consisting of alternating spatial domains with a phase shift of $\pi$ are observed within the $2: 1$ resonance regime, and three-phase patterns with spatial domains phase-shifted by $2 \pi / 3$ are observed within the 3:1 resonance regime [2, 16, 17]. The 4:1 resonance is more complicated. Adjacent spatial domains may differ in phase by either $\pi$ or $\pi / 2$. As a result the asymptotic patterns that develop can have four phases, two phases, or a mixture of two and four phases.

In this paper we explore pattern formation in the 4:1 resonance regimes. In Section II, we describe our experimental observations of four phase patterns in the 4:1 resonance band of the forced Belousov-Zhabotinsky reaction. We then present an analytical study of the 4:1 resonance [18, 19] in Section III. The study is based on a normal form, or amplitude equation, approach which is strictly valid only close to the Hopf bifurcation of the unforced oscillatory system. In order to test the analytical predictions and to study the behavior of forced systems far from the Hopf bifurcation, which is the case in the experiments, we conduct numerical studies of two reaction-diffusion models (the FitzHugh-Nagumo and Brusselator). We describe the models and results in Section IV. In Section $\mathrm{V}$ we discuss and compare the analytical and numerical results with the experimental observations.

\section{THE PERIODICALLY FORCED BELOUSOV-ZHABOTINSKY REACTION}

We use a light-sensitive form of the Belousov-Zhabotinsky (BZ) reaction, a chemical reaction system with oscillatory kinetics, to study the 4:1 subharmonic resonance patterns. In the experiments, the chemicals of the BZ system diffuse and react within a $0.4 \mathrm{~mm}$ thick porous membrane. The system is 


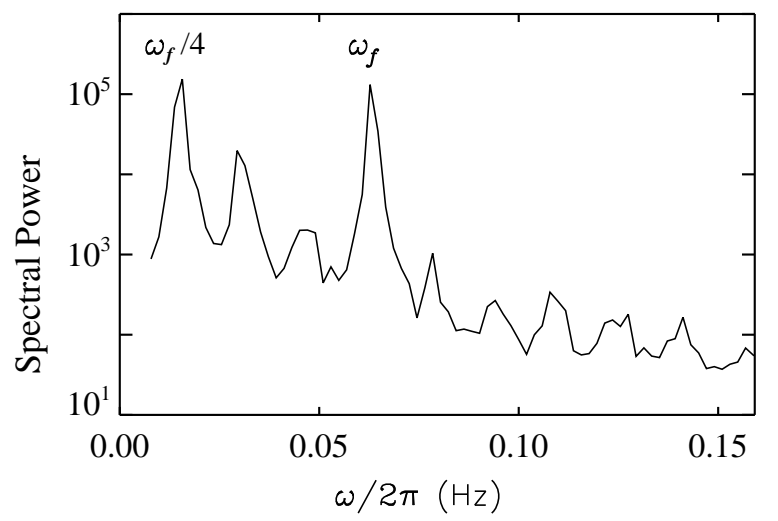

FIG. 1: The temporal power spectrum of a 4:1 resonant pattern from the BZ experiment for $I=426 \mathrm{~W} / \mathrm{m}^{2}$. The peak at $\omega / 2 \pi=0.0154$ $\mathrm{Hz}$ is the response at $\omega_{f} / 4$.

maintained in a non-equilibrium steady state by a continuous flow of fresh, well mixed reactant solutions [20] on either side of the thin membrane where the patterns form. The unforced pattern is a rotating spiral wave wave of ruthenium catalyst concentration.

We periodically force the system using spatially homogeneous square wave pulses of light with intensity $I$, where $I$ is the square of the forcing amplitude, and pulse frequency $\omega_{f}\left(\omega_{f} / 2 \pi\right.$ in $\left.\mathrm{Hz}\right)$. We choose the frequency $\omega_{f}$ to be approximately four times the natural frequency of the unforced oscillations.

To determine the temporal response of a pattern when it is periodically perturbed at a particular pair of $\left(I, \omega_{f}\right)$ parameter values we collect a time series of evenly sampled pattern snapshots; a $60 \times 60$ pixel region of the $640 \times 480$ pixel image. We sample at a rate of approximately 30 frames/oscillation and calculate the Fast Fourier Transform for the time series of each pixel. The power spectrum of each pixel is determined. An average over all pixels provides a power spectrum of a pattern, as shown in Fig. 1. The 4:1 resonant patterns exhibit a dominant peak at $\omega_{f} / 4$ in the power spectrum. Higher order harmonics are also present.

An example of a 4:1 resonant pattern observed in the experiments is shown in Fig. 2. The rotating four-phase spiral wave in Fig. 2(a) is the asymptotic state of the system. This image is a plot of the phase angle $\arg (a)$, where $a=a(x, y)$ is the complex Fourier amplitude associated with the $\omega_{f} / 4$ mode for each pixel $(x, y)$ in the pattern. The four domains (white, light gray, dark gray and black) correspond to the four phase states with oscillation phases that are shifted by $0, \pi / 2, \pi$, and $3 \pi / 2$ with respect to the forcing.

Fig. 2(b) is a different representation of the same data. In this case the response $a$ at $\omega_{f} / 4$ is plotted in the complex plane instead of the $x-y$ plane. This representation of the data allows us to see the distribution of the oscillation amplitude and phase at all pixels in the pattern. The four corners of the diamond shape in Fig. 2(b) are the four stable phase states. The edges of the diamond shape in Fig. 22(b) are formed from pixels at phase-fronts separating adjacent domains. The majority

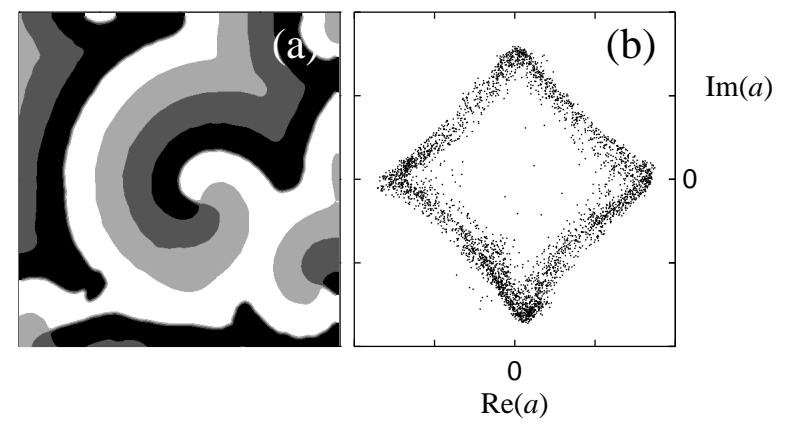

FIG. 2: A rotating four-phase spiral wave observed in the forced BZ reaction. (a) A $5.4 \times 5.4 \mathrm{~mm}^{2}$ region of a reactor image showing a 4:1 resonant spiral pattern. The white, light gray, dark gray and black domains represent the four phase states of the system. (b) A plot of the complex Fourier amplitude $a$ at $\omega_{f} / 4$ for each pixel of the pattern. The forcing intensity is $I=426 \mathrm{~W} / \mathrm{m}^{2}$ and the forcing frequency is $\omega_{f} / 2 \pi=0.062 \mathrm{~Hz}$. The data were filtered to isolate the response at $\omega_{f} / 4$ from the higher harmonics.

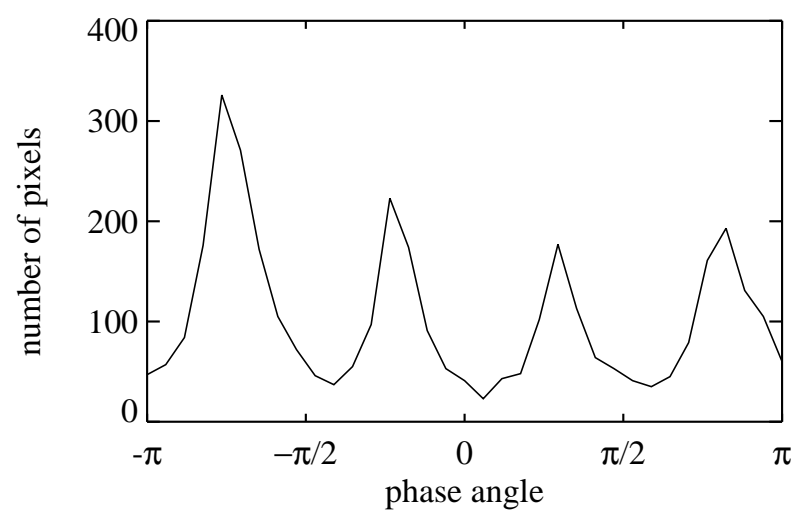

FIG. 3: A histogram showing the distribution of phase angles in the pattern in Fig. The four peaks indicate the high density of points in each of the four phase states.

of pixels in the pattern are in one of the four corner states as the histogram of phase angles in Fig. Billustrates.

Traveling four-phase patterns exist over the entire dynamic range of forcing intensity $I$ in the 4:1 resonance region. The range of forcing intensity is limited by a $I$-dependence of the reaction kinetics. As $I$ is increased, the reaction kinetics shifts from oscillatory to excitable.

\section{AN AMPLITUDE EQUATION FOR FORCED OSCILLATORY SYSTEMS}

We study the experimental observations shown in the previous section using a normal form equation for the amplitude of the $\omega_{f} / 4$ mode. Consider first an oscillatory system responding to the forcing at $\omega_{f} / n$ where $n$ is integer. We assume the system is near the onset of oscillations, i.e. close to a Hopf bifurcation. The set of dynamical fields $\mathbf{u}$ describing 
the spatio-temporal state of the system can be written as

$$
\mathbf{u}=\mathbf{u}_{\mathbf{0}} A \exp \left(i \omega_{f} t / n\right)+\text { c.c. }+\ldots,
$$

where $\mathbf{u}_{\mathbf{0}}$ is constant, $A$ is a slowly varying complex amplitude, and the ellipses denote other resonances with smaller contributions. The slow space and time evolution of the amplitude $A$ is described by the forced complex Ginzburg-Landau equation,

$$
\begin{aligned}
A_{\tau}= & (\mu+i \nu) A+(1+i \alpha) A_{z z}-(1-i \beta)|A|^{2} A \\
& +\gamma_{n} A^{*(n-1)},
\end{aligned}
$$

where $\mu$ is the distance from the Hopf bifurcation, $\nu$ is the detuning from the exact resonance, and $\gamma_{n}$ is the forcing amplitude.

For the special case $n=4$ (the 4:1 resonance) we can eliminate the parameter $\mu$ by rescaling time, space, and amplitude as $t=\mu \tau, x=\sqrt{\mu / 2} z$ and $B=A \sqrt{\mu}$ to obtain

$$
\begin{aligned}
B_{t}= & \left(1+i \nu_{0}\right) B+\frac{1}{2}(1+i \alpha) B_{x x}-(1-i \beta)|B|^{2} B \\
& +\gamma B^{* 3},
\end{aligned}
$$

where $\nu_{0}=\nu / \mu$. Equation (3) also applies to the 4:3 subharmonic resonance. This follows from symmetry considerations: the system is symmetric to discrete time translations $t \rightarrow t+\frac{2 \pi}{\omega_{f}}=t+\frac{3 \pi}{2 \omega}$. The amplitude equation must then be invariant under the transformation $B \rightarrow B \exp (3 \pi i / 2)$. The only forcing term satisfying this requirement to cubic order is $B^{* 3}$.

\section{A. Phase states and phase fronts}

Constant solutions of Eq. (3) indicate that the system is entrained to the forcing. There are four stable constant solutions to Eq. (3), each with the same amplitude but with different phases, $\arg (B)$, which correspond to the four stable phase states. Simple expressions for these solutions and exact forms for the front solutions connecting them in space are obtained from the gradient version of Eq. (3), where $\nu_{0}=\alpha=\beta=0$ :

$$
B_{t}=B+\frac{1}{2} B_{x x}-|B|^{2} B+\gamma B^{* 3} .
$$

The stable phase states (constant solutions) of Eq. (†) for $0<$ $\gamma<1$ are $\left(B_{1}, B_{2}, B_{3}, B_{4}\right)=(\lambda, i \lambda,-\lambda,-i \lambda)$ where $\lambda=$ $1 / \sqrt{1-\gamma}$. They are represented as solid circles in Fig. 4.

Front solutions connecting pairs of these states are of two types, fronts between states separated in phase by $\pi$ and fronts between states separated in phase by $\pi / 2$ (hereafter $\pi$-fronts and $\pi / 2$-fronts). The $\pi$-front solutions are

$$
\begin{aligned}
& B_{3 \rightarrow 1}=B_{1} \tanh x, \\
& B_{4 \rightarrow 2}=B_{2} \tanh x .
\end{aligned}
$$

For the particular parameter value $\gamma=1 / 3$ the $\pi / 2$-fronts have the simple forms

$$
B_{2 \rightarrow 1}=\frac{1}{2} \sqrt{\frac{3}{2}}[1+i+(1-i) \tanh x]
$$

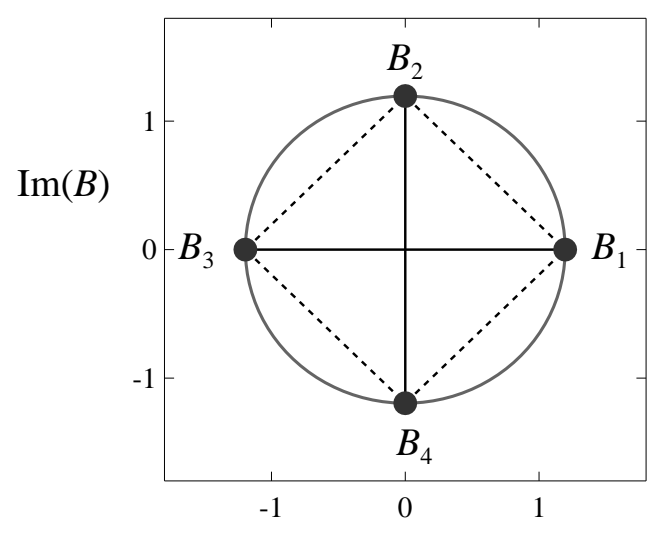

$\operatorname{Re}(B)$

FIG. 4: The four phase states (black dots) connected by phase-fronts in the forced complex Ginzburg-Landau equation (4). Two types of fronts between phase states are possible; the solid lines are $\pi$-fronts and the dashed lines are $\pi / 2$-fronts.

$$
\begin{aligned}
& B_{1 \rightarrow 4}=\frac{1}{2} \sqrt{\frac{3}{2}}[1-i-(1+i) \tanh x], \\
& B_{3 \rightarrow 2}=-B_{1 \rightarrow 4}, \\
& B_{4 \rightarrow 3}=-B_{2 \rightarrow 1} .
\end{aligned}
$$

Additional front solutions follow from the invariance of Eq. (4) under reflection, $x \rightarrow-x$.

Figure 4 shows these front solutions (parametrized by the spatial coordinate $x$ ) in the complex $B$ plane. For example, the $\pi$-front $B_{3 \rightarrow 1}$ is represented by the solid line connecting the state $B_{3}$ to the state $B_{1}$ as $x$ increases from $-\infty$ to $+\infty$. The $\pi / 2$-front $B_{2 \rightarrow 1}$ is represented by the dashed line connecting the state $B_{2}$ to the state $B_{1}$.

In the special case of the gradient system (4) all front solutions are stationary. The more general case with nongradient terms in Eq. (3) can be studied by perturbation theory when $\nu_{0}, \alpha$ and $\beta$ are small [19]. The results of this analysis show that the $\pi / 2$-fronts become propagating fronts while the $\pi$ fronts remain stationary.

Figure 5 shows a rotating four-phase spiral wave from a numerical solution of the two-dimensional version [21] of Eq. (3). The phase diagram in the complex $B$ plane, shown in Fig. 5(b), has four $\pi / 2$-fronts: $B_{1 \rightarrow 4}, B_{4 \rightarrow 3}, B_{3 \rightarrow 2}$ and $B_{2 \rightarrow 1}$. The amplitude $B$ corresponds to the complex Fourier amplitude $a$ measured in the experiment; the four-phase spiral pattern in Fig. 2 and the corresponding diamond-shape in the complex plane are predicted by the amplitude equation.

\section{B. A phase-front instability}

The existence of the stationary $\pi$-front solutions suggests that standing two-phase patterns similar to those found under 2:1 resonant conditions [2, 16] may be observed in the 4:1 resonant case provided the $\pi$-fronts are stable. Standing two-phase patterns have not been observed in experiments in 


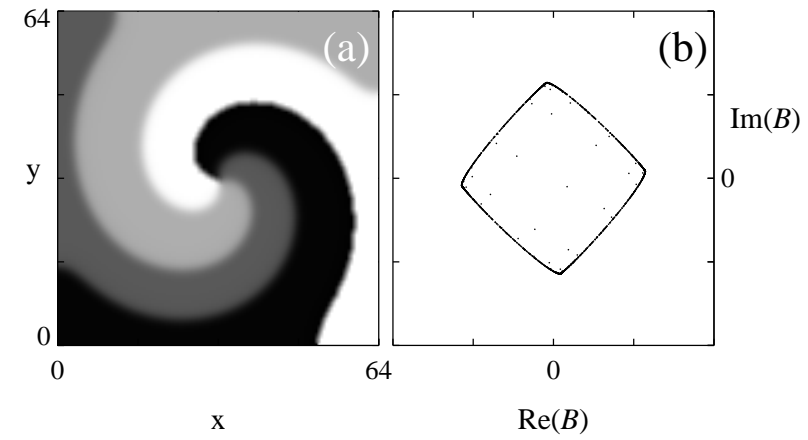

FIG. 5: A rotating four-phase spiral wave in the forced complex Ginzburg-Landau equation. (a) $\arg (B(x, y))$ in the $x-y$ plane. (b) $B(x, y)$ in the complex plane. Parameters: $\gamma=0.6, \nu_{0}=0.1$, $\alpha=\beta=0$.

the 4:1 resonance band so the stability of $\pi$-fronts becomes a question. Stability conditions for $\pi$-front solutions were studied in Refs. [18, 19]. The results are described below.

Consider the pair of $\pi / 2$-fronts shown in Fig. 6(a). They are separated by a distance $2 \chi$ and connect the phase states $B_{3}$ and $B_{1}$. For $\gamma \approx 1 / 3$, the solutions (6) are good approximations to $\pi / 2$-front solutions. The pair of fronts can be represented as

$$
B(x ; \zeta, \chi) \approx B_{3 \rightarrow 2}(x-\zeta+\chi)+B_{2 \rightarrow 1}(x-\zeta-\chi)-i \lambda,
$$

where $\zeta$ is their mean position. For large separation distances $(\chi>>1) B \approx B_{3 \rightarrow 2}$ when $x \approx \zeta-\chi$ and $B \approx B_{2 \rightarrow 1}$ when $x \approx \zeta+\chi$ and Eq. (7) represents a pair of isolated $\pi / 2$ fronts. When the distance between the pair decreases to zero $(\chi \rightarrow 0)$, then $B \approx B_{3 \rightarrow 1}$ and Eq. (7) approaches a $\pi$-front solution.

The stability of $\pi$-fronts is determined by the interaction between a pair of $\pi / 2$-fronts. Stable $\pi$-fronts are the result of an attractive $\pi / 2$-front interaction; the $\pi / 2$-fronts attract each other and the distance between them decreases to zero. A repulsive interaction implies unstable $\pi$-fronts. The potential $V(\chi)$ that governs this interaction,

$$
\dot{\chi}=-\frac{d V}{d \chi}
$$

is shown in Fig. 6(b) for various $\gamma$ values. The potential has a single maximum for $\gamma<\gamma_{c}=1 / 3$ which represents a repulsive interaction between $\pi / 2$-fronts and the instability of $\pi$-fronts. It has a single minimum for $\gamma>\gamma_{c}$ which indicates the attractive interaction between $\pi / 2$-fronts and the resulting stability of $\pi$-fronts. At $\gamma_{c}$ the potential is flat, $V=0$, for all $\chi$ values. At this parameter value, pairs of $\pi / 2$-fronts do not interact and there is a continuous family of front pair solutions with arbitrary separation distances, $2 \chi$, in Eq. (7). This degeneracy of solutions at the critical point $\gamma=\gamma_{c}$ is removed by adding higher order terms to the amplitude equation, as we discuss in Section IIID.

To summarize, stationary $\pi$-front solutions of Eq. (3) are stable for forcing amplitudes $\gamma>\gamma_{c}=1 / 3$. When $\gamma$ is
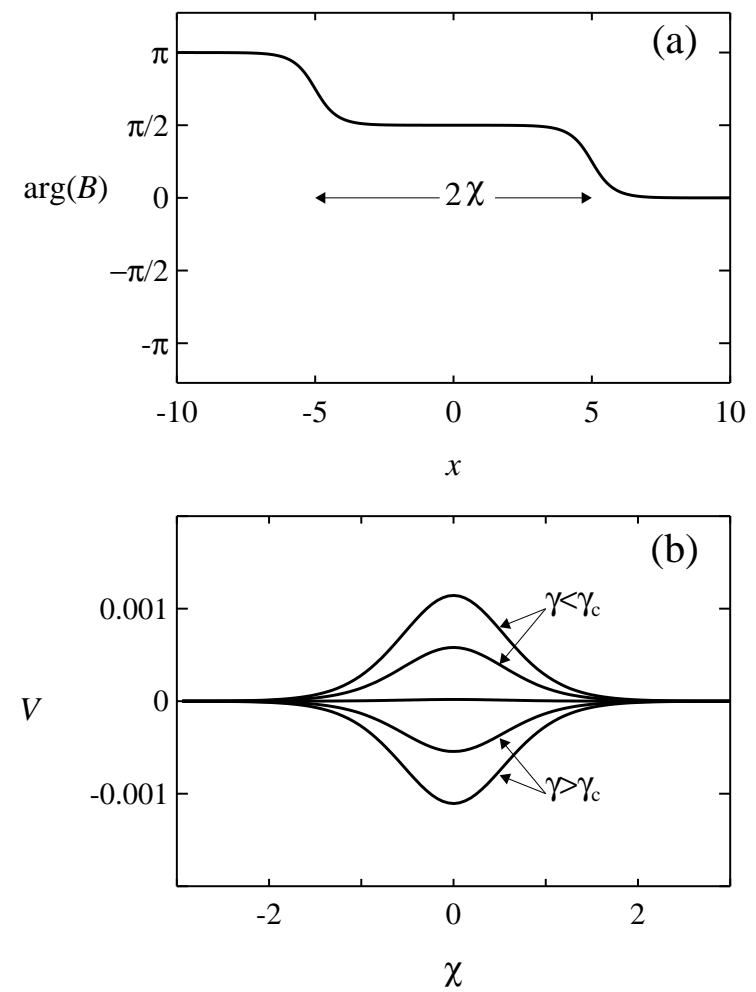

FIG. 6: (a) The phase, $\arg (B)$ of a pair of $\pi / 2$-fronts, $B_{3 \rightarrow 2}$, and $B_{2 \rightarrow 1}$. The distance between the two fronts is defined to be $2 \chi$. (b) The potential $V(\chi)$ describing the interaction between two $\pi / 2$ fronts. For $\gamma>\gamma_{c}$ the extremum at $\chi=0$ is a minimum and $\chi$ converges to 0 . For $\gamma<\gamma_{c}$ the extremum is a maximum and $\chi$ diverges to $\pm \infty$. At $\gamma=\gamma_{c}$ the potential is flat and there is no interaction between $\pi / 2$-fronts.

decreased past $\gamma_{c}, \pi$-fronts lose stability and split into pairs of propagating $\pi / 2$-fronts. The splitting process is shown in Fig. 7 where the $B_{3 \rightarrow 1} \pi$-front evolves into the pair of stable traveling $\pi / 2$-fronts, $B_{3 \rightarrow 2}$ and $B_{2 \rightarrow 1}$ when $\gamma<\gamma_{c}$. The parity symmetry $\chi \rightarrow-\chi$ makes evolution toward the pair $B_{1 \rightarrow 4}$ and $B_{4 \rightarrow 3}$ equally likely. The splitting occurs for forcing amplitudes arbitrarily close to $\gamma_{c}$, although in that case the time scale of this process becomes very long.

\section{Effects of the phase-front instability on pattern formation}

The stability of stationary $\pi$-fronts for $\gamma>\gamma_{c}$ suggests the predominance of standing two-phase patterns. These patterns involve alternating domains with oscillation phases shifted by $\pi$ with respect to one another. Domains shifted by $\pi / 2$ may exist as transients; the interactions between $\pi$-fronts and $\pi / 2$-fronts always produce $\pi / 2$-fronts which are stable but attract one another and coincide to form stationary $\pi$-fronts. Since the $\pi / 2$-fronts are traveling these transients are relatively short. For $\gamma<\gamma_{c}$ the interactions between the $\pi / 2$ fronts are repulsive. The $\pi$-fronts are unstable and split into pairs of traveling $\pi / 2$-fronts. As a result, traveling waves with 


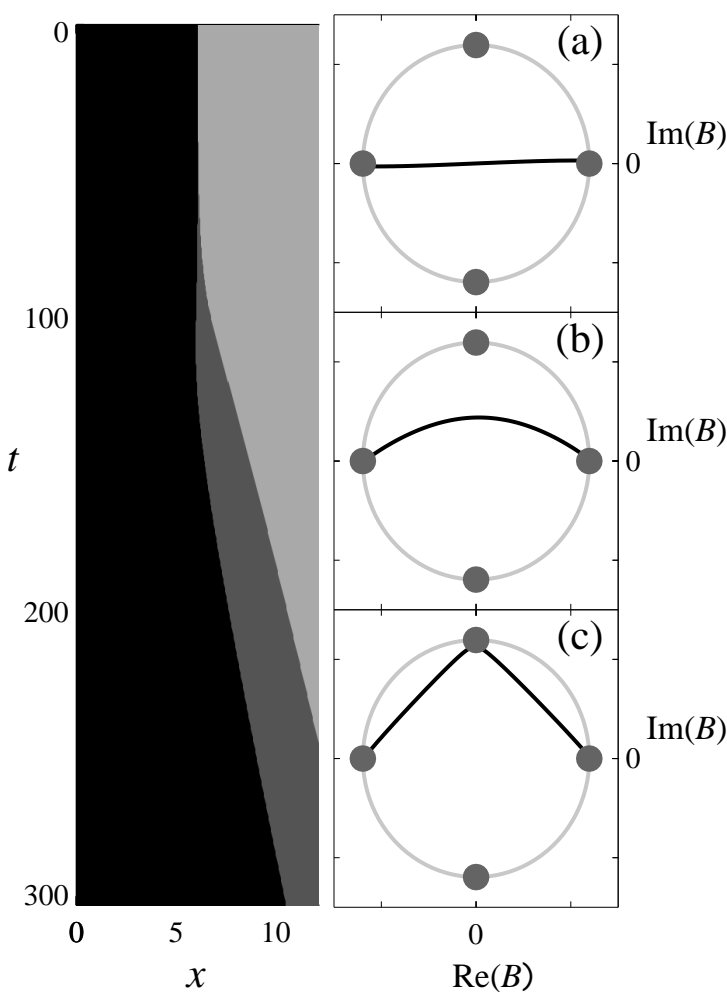

FIG. 7: An example of the phase-front instability in one space dimension. Left: The space-time plot (solutions of Eq. (3)) shows the splitting of an unstable $\pi$-front into a pair of traveling $\pi / 2$-fronts. The $\pi / 2$-front pairs enclose the dark gray domain that has an oscillation phase shifted by $\pi / 2$ with respect to the black and light gray domains. Right: Snapshots at times $t=0, t=100$, and $t=300$, showing the instability in the complex $B$ plane. Parameters in Eq. (3): $\nu_{0}=0.02, \gamma=0.3, \alpha=\beta=0$.

all four phase-states are the asymptotic pattern.

A typical two-dimensional traveling pattern involving all four phases is the four-phase spiral wave shown in Fig. 2 or in Fig. 5. Figure 8 shows the effect of the phase-front instability on a four-phase spiral wave. The initial spiral wave (Fig. 8(a)) was obtained by solving a two-dimensional version of Eq. (3) for $\gamma<\gamma_{c}$. The following three frames (Fig. \&(b)(d)) are snapshots showing the evolution of the initial fourphase spiral wave into a standing two-phase pattern after $\gamma$ is increased above $\gamma_{c}$. The evolution begins at the spiral core where the attractive interactions between pairs of $\pi / 2$-fronts are the strongest. The coalescence of $\pi / 2$-fronts leaves behind a stationary $\pi$-front which grows in length until no $\pi / 2$-fronts are left, as is evident by the single line in the complex $B$ plane shown in Fig. 8(d).

\section{Higher order terms in the amplitude equation}

From the analysis of Eq. (3) we have shown that two-phase patterns must be standing and four-phase patterns must be traveling. The analysis of the equation with higher order con-

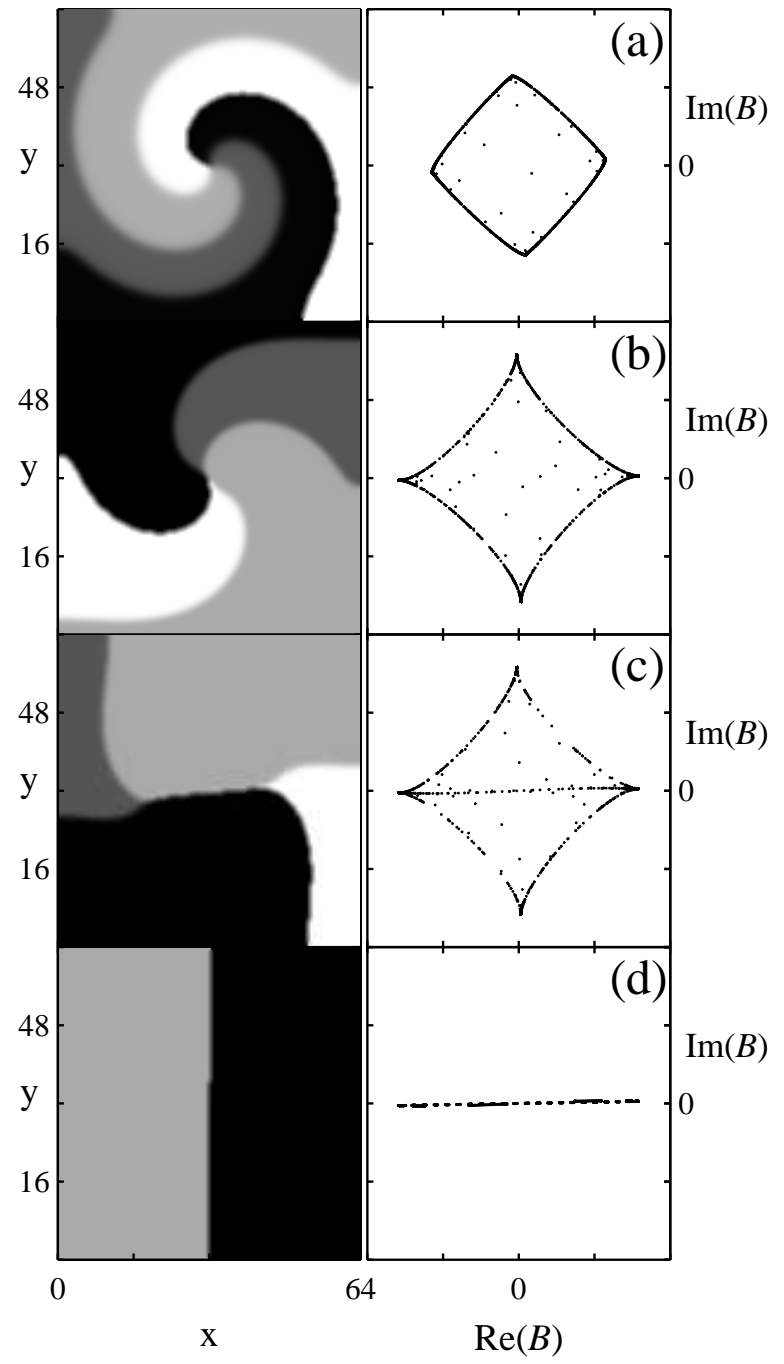

FIG. 8: Numerical solution of a two-dimensional version of Eq. (3) showing the evolution of a rotating four-phase spiral-wave into a standing two-phase pattern when $\gamma$ is increased above $\gamma_{c}$. The frames on the left show $\arg (B)$ in the $x-y$ plane. The frames on the right show the complex $B$ plane. (a) The initial four-phase spiral wave (computed with $\gamma<\gamma_{c}$ ). (b) The spiral core, a 4-point vertex, splits into two 3-point vertices connected by a $\pi$-front. (c) A two-phase pattern develops as the 3-point vertices further separate. (d) The final standing two-phase pattern. Parameters: $\gamma=0.6, \nu_{0}=0.1$, $\alpha=\beta=0, \gamma_{c} \approx 1 / 3$.

tributions suggests the possible existence of a small $\gamma$ range, of order $\mu \ll 1$, surrounding $\gamma_{c}$ where slowly traveling twophase patterns exist.

The higher order contributions to Eq. (3), such as $|B|^{4} B$, or $|B|^{2} B_{x x}$, lift the degeneracy of the instability. Figure 9 shows two possible scenarios for the front interaction potential $V$ when higher order contributions to Eq. (3) are included (both scenarios lift the degeneracy of the phase-front instabil- 
ity). In one case, shown in Fig. $9($ a), the stationary $\pi$-front loses stability to a pair of counter-propagating $\pi$-fronts in a pitchfork bifurcation which leads to double-minimum potential. This scenario is a nonequilibrium Ising-Bloch pitchfork bifurcation of $\pi$-fronts like the one found in the 2:1 resonance case [22] and in other bistable systems [13, 23, 24, 25]. It leads to slow traveling two-phase patterns in the range where $\gamma$ is near $\gamma_{c}$. In the scenario shown in Fig. 9 (b), the stationary $\pi$-front loses stability via a subcritical bifurcation which leads to double-maximum potential. In this case there is a range of stable $\pi$-fronts coexisting with pairs of separated $\pi / 2$-fronts. This allows the possibility of patterns containing both $\pi$-fronts and $\pi / 2$-fronts. Beyond this range the potential has a single maximum and $\pi$-fronts split into pairs of $\pi / 2$-fronts. Both scenarios persist over a range of $\gamma$ of order $\mu$, the distance from the Hopf bifurcation.



FIG. 9: The degeneracy of the potential $V(\chi)$ at $\gamma=\gamma_{c}$ is broken by adding higher order terms to (3). In the intermediate range of $\gamma \approx \gamma_{c}$ two scenarios are possible as $\gamma$ is decreased through the bifurcation: (a) The $\chi=0$ solution loses stability in a pitchfork bifurcation at $\gamma_{c}$ to a pair of solutions that move to $\pm \infty$. (b) The $\chi=0$ solution remains stable while the $\chi= \pm \infty$ solutions acquire stability and lose stability only below $\gamma_{c}$. In both cases the deformations from a single minimum to a single maximum occur within a small range of $\gamma$ of order $\mu \ll 1$.

\section{NUMERICAL SOLUTIONS OF PERIODICALLY FORCED REACTION-DIFFUSION MODELS}

The amplitude equation analysis predicts the existence of a phase-front instability near the Hopf bifurcation and hints at possible modifications of the instability as the distance from the Hopf bifurcation is increased. Our objectives in this sec- tion are to test the existence of the instability in reactiondiffusion models and to use the models to examine how the instability is modified far from the Hopf bifurcation.

\section{A. The FitzHugh-Nagumo model}

We study a periodically forced version of the FitzHughNagumo equations

$$
\begin{aligned}
& u_{t}=u-\left(1+\Gamma \cos \omega_{f} t\right) u^{3}-v+\nabla^{2} u \\
& v_{t}=\epsilon\left(u-a_{1} v\right)+\delta \nabla^{2} v
\end{aligned}
$$

The unforced model is obtained by setting $\Gamma=0$. The uniform state $(u, v)=(0,0)$ undergoes a Hopf bifurcation as $\epsilon$ is decreased past $\epsilon_{c}=1 / a_{1}$. The Hopf frequency is $\omega_{H}=\sqrt{\epsilon_{c}-1}$ and the distance from the Hopf bifurcation is measured by $\mu=\left(\epsilon_{c}-\epsilon\right) / \epsilon_{c}$.

We compute the numerical solutions of Eq. (9) in the 4:1 resonance band $\left(\omega_{f} \approx 4 \omega_{H}\right)$ and close to the Hopf bifurcation $(\mu \ll 1)$. Close to the Hopf bifurcation the amplitude equation analysis applies. We expect to find a critical value of the forcing amplitude $\Gamma_{c}$ corresponding to the phase-front instability point $\gamma_{c}$ in the amplitude equation. For the FitzHughNagumo equations this $\Gamma_{c}$ will, in general, depend on the parameters $\epsilon, \delta, a_{1}$, and $\omega_{f}$. In the following we fix $a_{1}=1 / 2$, $\delta=0, \omega_{f}=4$ and only vary $\epsilon$ (the parameter that controls the distance $\mu$ to the Hopf bifurcation) and the forcing amplitude $\Gamma$.

Close to the Hopf bifurcation we find stable stationary $\pi$ fronts for forcing amplitudes $\Gamma>\Gamma_{c}$. Below $\Gamma_{c}$, stationary $\pi$ fronts are unstable and split into pairs of $\pi / 2$-fronts. Figure 10 illustrates this in numerical solution of a one-dimensional version of Eq. (9). An stable $\pi$-front pattern is generated from random initial conditions with $\Gamma>\Gamma_{c}$. At $t=0 \Gamma$ is decreased below $\Gamma_{c}$; the $\pi$-front becomes unstable and splits into a pair of traveling $\pi / 2$-fronts.

The numerically computed $\Gamma_{c}$ for the solution in Fig. 10 is $\Gamma_{c} \approx 2.15$. Since $\Gamma_{c}$ is a function of the parameters in Eq. (9), we define a new parameter $\eta=\left(\Gamma_{c}-\Gamma\right) / \Gamma_{c}$ that measures the distance from the phase-front instability point. In Fig. 10, $\eta \approx 0.012$ indicating that we are just beyond the critical point.

Farther from the Hopf bifurcation we find that the phasefront instability still exists. Figure 11 shows the the evolution of an initial unstable stationary $\pi$-front with parameters chosen so the system is far from the Hopf bifurcation but at the same distance, $\eta \approx 0.012$, from the phase-front instability. The asymptotic solution is a slowly propagating $\pi$-front, in contrast to a pair of separated $\pi / 2$-fronts that develop close to the Hopf bifurcation (see Fig. 10). The range of forcing amplitudes near $\Gamma_{c}$ over which these traveling $\pi$-fronts exist increases with $\mu$. At smaller forcing amplitudes, below the range of traveling $\pi$-fronts, $\pi$-fronts split into pairs of $\pi / 2$ fronts and four phase traveling patterns prevail.

In two dimensions the typical traveling wave pattern for $\Gamma<\Gamma_{c}$ is a rotating four-phase spiral wave. Figure 12 (a) shows a stable four-phase spiral wave generated from random initial conditions. Using this spiral as an initial condition, we 


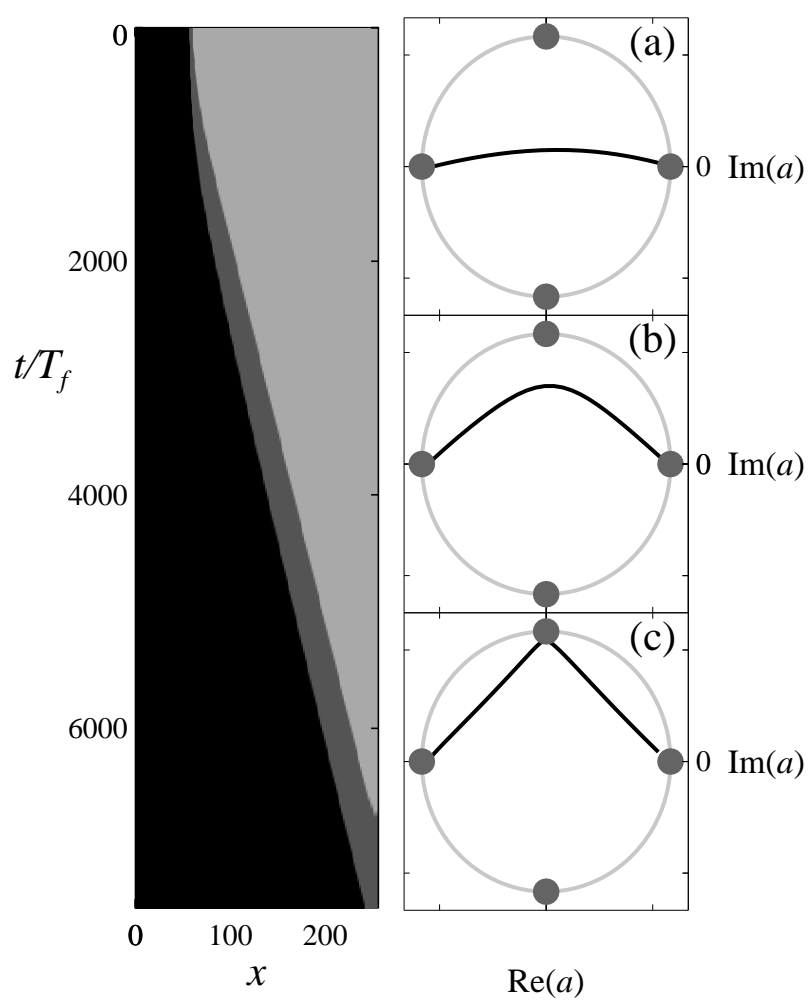

FIG. 10: The phase-front instability in the 4:1 resonance of the forced FitzHugh-Nagumo model close to the Hopf bifurcation. Left: a space-time plot of $\arg (a)$ where $a$ is the complex Fourier coefficient of the 4:1 response (equivalent to $A$ in Eq. (2)). At $t=0$ the forcing amplitude was decreased below $\Gamma_{c}$. The initial standing $\pi$ front becomes unstable and splits into a pair of traveling $\pi / 2$-fronts. The $\pi / 2$-fronts separate the black, dark gray, and light gray domains where the oscillation phase is shifted successively by $\pi / 2$. Right: The same data depicted in the complex $a$ plane at three successive times, $t=0, t=560 T_{f}$, and $t=4160 T_{f}$ where $T_{f}=2 \pi / \omega_{f}$. (a) The initial standing $\pi$-front is unstable. (b) The front develops an intermediate phase. (c) Two $\pi / 2$ fronts are formed. Parameters: $a_{1}=0.5, \epsilon=1.95, \delta=0, \Gamma=2.0, \omega_{f}=4.0$, and $\mu=0.025$. The phase-front instability point is $\Gamma_{c} \approx 2.15$ and $\eta \approx 0.012$.

increase $\Gamma$ above $\Gamma_{c}$ and the system evolves into a two-phase standing pattern. Figures 12 (b)-(d) show the transition. Since the $\pi / 2$ fronts are attracting the spiral is unstable and two of the four phase domains shrink until a standing two-phase pattern remains.

The numerical solutions of the forced FitzHugh-Nagumo equations support the predictions of the amplitude equation analysis. Close to the Hopf bifurcation, the phase-front instability is found (compare Fig. 77 with Fig. 10 and Fig. 8 with Fig. 12). Far from the Hopf bifurcation the instability persists. The effects of higher order terms in the amplitude equation are valid even far from the Hopf bifurcation $(\mu=0.25)$; the phase-front instability near the Hopf bifurcation (as $\mu \rightarrow 0$ ) turns into an Ising-Bloch pitchfork bifurcation. Stationary $\pi$ fronts bifurcate to traveling $\pi$-fronts and not $\pi / 2$-fronts.

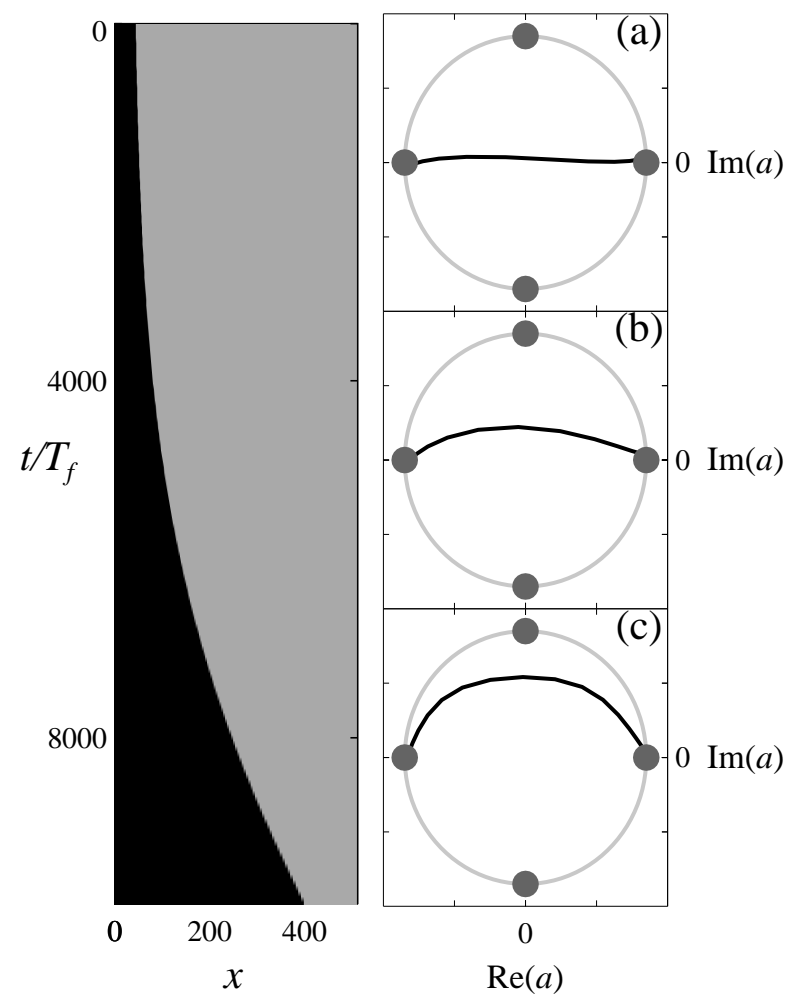

FIG. 11: The phase-front instability in the 4:1 resonance of the forced FitzHugh-Nagumo model far from the Hopf bifurcation. Left: a space-time plot of $\arg (a)$. At $t=0$ the forcing amplitude was decreased below $\Gamma_{c}$. The initial standing $\pi$-front is unstable and and starts traveling to the right. In this case, no intermediate phase develops. Right: The same data depicted in the complex $a$ plane at three successive times, $t=0, t=3000 T_{f}$, and $t=6000 T_{f}$ where $T_{f}=2 \pi / \omega_{f}$. (a) The initial standing $\pi$-front. (b) The standing $\pi$-front is unstable and begins to travel. (c) The asymptotic pattern is a traveling $\pi$-front. Parameters: $a_{1}=0.5, \epsilon=1.5, \delta=0$, $\Gamma=1.585, \omega_{f}=4.0$, and $\mu=0.25$. The phase-front instability point is $\Gamma_{c} \approx 1.605$ and $\eta \approx 0.012$.

\section{B. The Brusselator model}

We tested the transition from four-phase traveling waves to two-phase standing waves using another reaction-diffusion model, the forced Brusselator,

$$
\begin{aligned}
& u_{t}=c-d u+\left[1+\Gamma \cos \omega_{f} t\right] u^{2} v+\nabla^{2} u, \\
& v_{t}=d u-u^{2} v+\delta \nabla^{2} v .
\end{aligned}
$$

The unforced Brusselator, obtained by setting $\Gamma=0$, has a stationary uniform state $(u, v)=(c, d / c)$ which undergoes a Hopf bifurcation as $d$ is increased past $d_{c}=1+c^{2}$. The Hopf frequency is $\omega_{H}=c$ and the distance from the Hopf bifurcation is measured by $\mu=\left(d-d_{c}\right) / d_{c}$.

We studied Eq. (10) in the 4:1 resonance band using a numerical partial differential equation solver [26, 27]. We found that below a critical forcing amplitude $\Gamma_{c}$ the solutions are rotating four-phase spiral waves consisting of $\pi / 2$-fronts (see Fig. 13(a)). The four-phase spiral wave was generated by one 


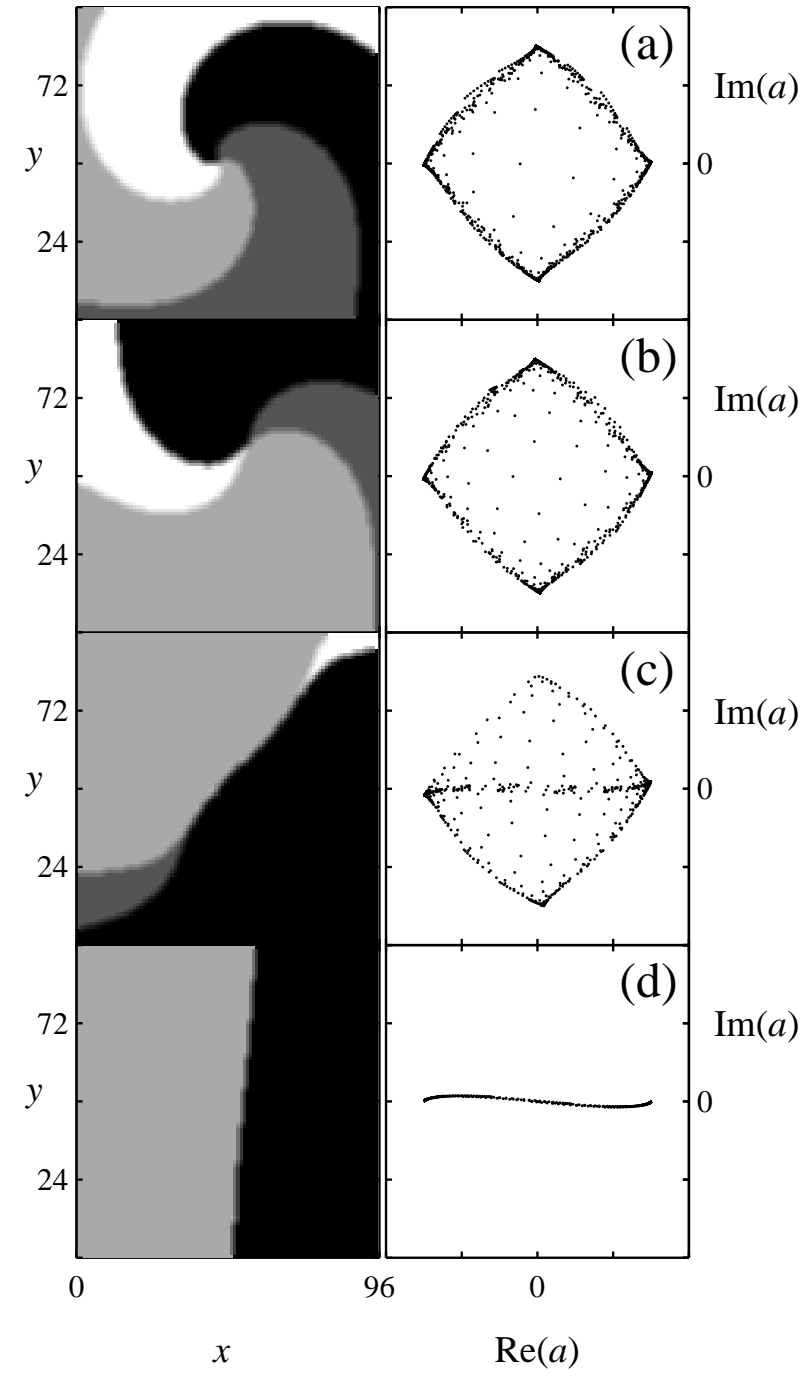

FIG. 12: Numerical solution of the forced FitzHugh-Nagumo equations (9) in 4:1 resonance shown at four successive times $t=0$, $t=11600 T_{f}, t=13600 T_{f}$, and $t=15600 T_{f}$ where $T_{f}=2 \pi / \omega_{f}$. The frames on the left show $\arg (a)$ in the $x-y$ plane. The frames on the right show the complex $a$ plane. (a) The initial spiral wave of four phases separated by $\pi / 2$-fronts is computed with $\Gamma<\Gamma_{c}$. (b) When $\Gamma$ is increased above $\Gamma_{c}$ two pairs of $\pi / 2$-fronts begin to attract one another. (c) As the $\pi / 2$-fronts attract they collapse into a stationary $\pi$-front which grows in length. (d) The final pattern is two phase domains separated by a stationary $\pi$-front. Parameters: $a_{1}=0.5, \epsilon=1.5, \delta=0, \Gamma=2.5$, and $\omega_{f}=4.0$.

of two following initial conditions: a spiral wave computed from the unforced $(\Gamma=0)$ Brusselator equations, or the linear functions

$$
\begin{aligned}
& u(x, y)=\quad y / L, \quad 0 \leq y \leq L, \\
& v(x, y)=-2 x / L+4 \quad 0 \leq x \leq L \text {, }
\end{aligned}
$$

where $L=632.5$.
Above $\Gamma_{c}$ pairs of $\pi / 2$ fronts attract each other and the core of the spiral evolves into an expanding $\pi$-front. Figures 13.(b)(d) illustrate this process. When the $\pi / 2$ fronts disappear, the resulting asymptotic pattern is two states separated by a stationary $\pi$-front. The transition from a four-phase spiral wave to a two-phase stationary pattern, as in the amplitude equation model and the FitzHugh-Nagumo model, indicates the existence of the phase-front instability in the Brusslator model.



FIG. 13: Numerical solutions of the forced Brusselator model (10) showing snapshots at $t=0, t=748 T_{f}, t=1000 T_{f}$, and $t=$ $5544 T_{f}$ where $T_{f}=2 \pi / \omega_{f}$. The rotating four-phase spiral wave, computed with $\Gamma<\Gamma_{c}(\Gamma=0.11)$, transforms into a standing twophase pattern after $\Gamma$ is increased past $\Gamma_{c}(\Gamma=0.13)$. The frames in the left column show $\arg (a)$ in the $x-y$ plane where $a$ is the complex Fourier coefficient of the 4:1 mode. The right column shows the same data in the complex $a$ plane. Parameters: $c=0.5, d=1.5$, $\delta=5.0, \omega_{f}=1.69$, and $\mu=0.20$. The numerical solution grid was $128 \times 128$ points. 


\section{CONCLUSIONS}

We studied 4:1 resonant patterns in Belousov-Zhabotinsky chemical experiments, in an amplitude equation for forced oscillatory systems (the forced complex Ginzburg-Landau equation), and in forced FitzHugh-Nagumo and Brusselator reaction-diffusion models. At low forcing amplitudes all of these systems exhibit traveling four-phase patterns.

An analysis of a forced complex Ginzburg-Landau equation, derivable from periodically forced reaction-diffusion systems near a Hopf bifurcation, predicts traveling four-phase patterns at low forcing amplitude and standing two-phase patterns at high forcing amplitude. The transition mechanism between these two patterns is a degenerate phase-front instability where a stationary $\pi$-front splits into a pair of traveling $\pi / 2$-fronts. We derived an interaction potential between $\pi / 2$ fronts that describes the instability as a change from repulsive to attractive $\pi / 2$-front interactions. We investigated the behavior of the instability near the critical point where higher order terms in the amplitude equation become important. We found that these terms lift the degeneracy of the instability and introduce a narrow intermediate regime. In this regime we found both slowly traveling $\pi$-fronts and the coexistence of stable stationary $\pi$-fronts and repelling pairs of $\pi / 2$-fronts.

We further investigated this phase-front instability using the FitzHugh-Nagumo and the Brusselator reaction-diffusion models. These models exhibit the instability even far from the Hopf bifurcation where the amplitude equation is not known to be valid. Near the Hopf bifurcation the instability, at $\Gamma_{c}$, separates patterns of stationary $\pi$-fronts from patterns of traveling $\pi / 2$-fronts. In two dimensions, a rotating four-phase spiral wave evolves into a two-phase standing pattern when $\Gamma$ is increased past $\Gamma_{c}$. In the FitzHugh-Nagumo model we found, far from the Hopf bifurcation, an intermediate range near $\Gamma_{c}$ where traveling $\pi$ front patterns were observed. These numerical results are in full agreement with the theoretical predictions based on the amplitude equation.

The standing two-phase patterns found in the amplitude equation and in the FitzHugh-Nagumo and Brusselator models were not observed in the experiments, which were conducted far from the Hopf bifurcation. However, the existence of the phase-front instability far from the Hopf bifurcation was found in the numerical studies of the FitzHugh-Nagumo and Brusselator models. We conclude that the large distance from the Hopf bifurcation does not explain the absence of standing two-phase patterns in the experiments. A more likely explanation is the limited dynamic range of the forcing amplitude in the experiments. Experiments show that the dynamics of the $\mathrm{BZ}$ reaction are $\gamma$-dependent; as the forcing amplitude is increased, the dynamics undergo a transition from oscillatory to excitable kinetics. The excitable kinetics are not described by the amplitude equation or by the reaction-diffusion models in the parameter ranges we studied.

\section{Acknowledgments}

We acknowledge the support of the Engineering Research Program of the Office of Basic Energy Sciences of the U.S. Department of Energy. Additional support was provided by the ASCI project B347883 through the Lawrence Berkeley National Lab; the Robert A. Welch Foundation; grant No. 98-00129 from the United States - Israel Binational Science Foundation; and by the Department of Energy, under contract W-7405-ENG-36.
* Electronic address: aric@ lanl.gov

$\dagger$ Electronic address: ehud@bgumail.bgu.ac.i1

[1] R. Kapral and K. Showalter, eds., Chemical Waves and Patterns (Kluwer Academic Publishers, Dordrect, 1994).

[2] V. Petrov, Q. Ouyang, and H. L. Swinney, Nature 388, 655 (August 1997).

[3] P. B. Umbanhowar, F. Melo, and H. L. Swinney, Nature 382, 793 (1996).

[4] P. Kolodner, D. Bensimmon, and C. M. Surko, Phys. Rev. Lett. 60, 1723 (1988).

[5] J. E. Pearson, Science 261, 189 (July 1993).

[6] K. J. Lee and H. L. Swinney, Phys. Rev. E 51, 1899 (1995).

[7] A. Hagberg and E. Meron, Phys. Rev. Lett. 72, 2494 (1994).

[8] A. Hagberg and E. Meron, Chaos 4, 477 (1994).

[9] R. E. Goldstein, D. J. Muraki, and D. M. Petrich, Phys. Rev. E 53, 3933 (1996).

[10] K. J. Lee, W. D. McCormick, Q. Ouyang, and H. L. Swinney, Science 261, 192 (1993).

[11] G. Haas, M. Bär, I. G. Kevrekidis, P. B. Rasmussen, H.-H. Rotermund, and G. Ertl, Phys. Rev. Lett. 75, 3560 (1995).

[12] K. B. Migler and R. B. Meyer, Physica D 71, 412 (1994).

[13] T. Frisch, S. Rica, P. Coullet, and J. M. Gilli, Phys. Rev. Lett. 72, 1471 (1994).

[14] T. Frisch and J. M. Gilli, J. Phys. II France 5, 561 (1995).
[15] P. Coullet and K. Emilsson, Physica D 61, 119 (1992).

[16] A. L. Lin, M. Bertram, K. Martinez, H. L. Swinney, A. Ardelea, and G. F. Carey (1999), to appear in Phys. Rev. Lett.

[17] A. L. Lin, V. Petrov, A. Ardelea, G. F. Carey, and H. L. Swinney (1999), unpublished.

[18] C. Elphick, A. Hagberg, and E. Meron, Phys. Rev. Lett. 80, 5007 (1998).

[19] C. Elphick, A. Hagberg, and E. Meron, Phys. Rev. E 59, 5285 (1999).

[20] The chemical concentrations in the two chemical reserviors separated by the porous membrane were, Reservior I: $0.22 \mathrm{M}$ malonic acid, $0.2 \mathrm{M}$ sodium bromide, $0.264 \mathrm{M}$ potassium bromate, $0.8 \mathrm{M}$ sulfuric acid; Reservior II: $0.184 \mathrm{M}$ potassium bromate, $1 \times 10^{-3} \mathrm{M}$ Tris(2,2'bipyridyl)dichlororuthenium(II)hexahydrate, $0.8 \mathrm{M}$ sulfuric acid. Each reservior volume is $8.3 \mathrm{ml}$ and the flow rate of chemicals through Reservior I was $20 \mathrm{ml} / \mathrm{hr}$ while through Reservior II it was $5 \mathrm{ml} / \mathrm{hr}$. Chemicals were premixed before entering each reservior; a $10 \mathrm{ml}$ premixer and a $0.5 \mathrm{ml}$ premixer fed Reservior I and II, respectively. The experiments were conducted at room temperature.

[21] $B_{t}=\left(1+i \nu_{0}\right) B+\frac{1}{2}(1+i \alpha) \nabla^{2} B-(1-i \beta)|B|^{2} B+\gamma_{4} B^{* 3}$.

[22] P. Coullet, J. Lega, B. Houchmanzadeh, and J. Lajzerowicz, Phys. Rev. Lett. 65, 1352 (1990). 
[23] H. Ikeda, M. Mimura, and Y. Nishiura, Nonl. Anal. TMA 13, 507 (1989).

[24] A. Hagberg and E. Meron, Nonlinearity 7, 805 (1994).

[25] M. Bode, A. Reuter, R. Schmeling, and H.-G. Purwins, Phys. Lett. A 185, 70 (1994).
[26] A. L. Pardhanani and G. F. Carey, J. Comp. Appl. Math 74, 295 (1996).

[27] A. Ardelea, A. Pardhanani, G. F. Carey, M. Bertram, A. L. Lin, and H. L. Swinney (2000), unpublished. 